\title{
ARTICLES
}

\section{Phonon instabilities in high-pressure bec-fcc and the isostructural fec-fec phase transitions of Cs}

\author{
Jianjun Xie and S. P. Chen \\ Theoretical Division, Los Alamos National Laboratory, Los Alamos, New Mexico 87545 \\ John S. Tse, Dennis D. Klug, Zhiqiang Li, and Kentaro Uehara \\ Steacie Institute for Molecular Science, National Research Council of Canada, Ottawa, Ontario, Canada K1A OR6 \\ L. G. Wang \\ Fritz-Haber-Institut der Max-Planck-Gesellschaft, Faradayweg 4-6, D-14195 Berlin-Dahlem, Germany
}

(Received 27 January 2000)

\begin{abstract}
The phonon instabilities of cesium related to its pressure-induced bcc-to-fcc ${ }^{(1)}-$ to- $_{\text {fcc }}{ }^{(2)}$ phase transitions are studied using the density-functional perturbation theory. It is found that at low pressure, both the bcc and fcc $\mathrm{Cs}$ are dynamically stable, but fcc is thermodynamically metastable. The high-pressure phase transitions of Cs from bcc to $\mathrm{fcc}^{(1)}$ and from $\mathrm{fcc}^{(1)}$ to $\mathrm{fcc}^{(2)}$ are related to the phonon instabilities of the long-wavelength acoustic modes resulted from the negative tetragonal shear elastic constant $C^{\prime}=\frac{1}{2}\left(C_{11}-C_{12}\right)$. During the $\mathrm{fcc}^{(1)}$-to-fcc ${ }^{(2)}$ phase transition, all the phonon modes become soft, making it the most striking feature of this isostructural phase transition.
\end{abstract}

\section{INTRODUCTION}

Cesium metal exhibits an unusual sequence of phase transitions under pressure. ${ }^{1-6}$ The structure of ground-state Cs (Cs-I) at ambient pressure is body-centered cubic (bcc). At $2.37 \mathrm{GPa}$ and $V / V_{0}=0.63$, where $V_{0}$ is the equilibrium volume at zero pressure, its crystal structure changes to facecentered cubic (fcc) with a small reduction of volume. ${ }^{1}$ Near $4.2 \mathrm{GPa}$ and $V / V_{0}=0.455$, the high-pressure $\mathrm{fcc}^{(1)}$ phase (Cs-II) undergoes a first-order isostructural transition ${ }^{3}$ to another $\mathrm{fcc}^{(2)}$ phase (Cs-III) with a large volume change $(-9 \%)$. At $4.4 \mathrm{GPa}$, there is another transition to the tetragonal Cs-IV phase. ${ }^{6} \mathrm{Cs}-\mathrm{V}$ phase is also reported near $12 \mathrm{GPa}$ recently. ${ }^{7}$

Theoretical calculations have focused mainly on the changes of the electronic structures induced from external pressures. It has been found that the $6 s$ valence electron undergoes a transfer to a more localized $5 d$-like state ${ }^{8-10}$ at high pressure due to the downward motion of the $d$ bands in the vicinity of the Fermi level. In this paper, we present a different approach, i.e., a lattice dynamics study, on the bcc-to-fcc ${ }^{(1)}-$ to-fcc $^{(2)}$ phase transition. It is known that dynamic instabilities or anomalous softening are often related to the structural phase transitions. ${ }^{11-14}$ These dynamic instabilities may, for example, occur for wave vectors at the boundary of the first Brillouin zone (BZ), e.g., the softening of an acoustic phonon at $M$ point of the first $\mathrm{BZ}$ in the cubicto-orthorhombic transition of $\mathrm{CsCl} .{ }^{11}$ The instability may also occur for wave vectors well into the first BZ, as is exemplified for many metals in the bcc structure by the longitudinal phonon mode at $\mathbf{q}=\left[\frac{2}{3} \frac{2}{3} \frac{2}{3}\right]$ (Ref. 13) transforming the bcc phase into a $\omega$ phase for sufficiently large phonon amplitude. In other cases, the phonon instability occurs for wave vectors close to the center of the first $\mathrm{BZ},{ }^{14}$ i.e., $\mathbf{q}$ $\rightarrow 0$. This instability results from a negative elastic constant, often related to the martensitic transformation between the bcc and fcc structures. In this paper, we present a systematic study on the phonon structure of Cs as a function of pressure. We find that the structural phase transition of Cs from bcc to $\mathrm{fcc}^{(1)}$ to another $\mathrm{fcc}^{(2)}$ is related to the phonon instabilities at the center of the first $\mathrm{BZ}$ at the transition pressure.

\section{METHOD}

The total energy calculations are based on the densityfunctional theory (DFT) in the local-density approximation. Different computational approaches have been employed, i.e., pseudopotential plane-wave (PS-PW) method, ${ }^{11}$ fullpotential linear-augmented plane-wave (FP-LAPW) method, ${ }^{15}$ and full-potential linear muffin-tin orbital (FPLMTO) method. ${ }^{16}$ In the PS-PW calculations, the wave function is expanded by a plane-wave basis set with a kinetic energy cutoff of $25 \mathrm{Ry}$. Sums over occupied electronic states are performed by the Gaussian-smearing special-point technique ${ }^{17,18}$ with a smearing width of $50 \mathrm{mRy}$, on a 10 $\times 10 \times 10$ grid in the first BZ. The interaction between the valence electrons and the atomic core is described by the norm-conserving pseudopotentials. In a similar manner as described in the previous work, ${ }^{11}$ the $5 s, 5 p$, and $6 s$ electrons of $\mathrm{Cs}$ are treated as valence electrons. The total energy calculations (lattice constant, bulk modulus, and pressurevolume relationship) have been checked by the FP-LAPW method with a kinetic energy cutoff of $10 \mathrm{Ry}$ and $10 \times 10$ $\times 10$ k-point grid in the first BZ.

The phonon frequencies are calculated using densityfunctional perturbation theory ${ }^{19}$ on a $4 \times 4 \times 4$ regular mesh 


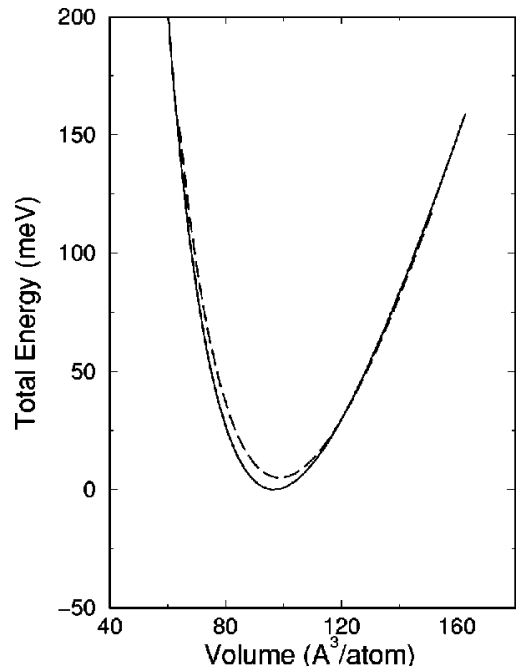

FIG. 1. The total energies of $\mathrm{Cs}$ as a function of volume computed from the PW-PS method. The solid line is for the bcc Cs. The dashed line is for the fcc Cs.

and Fourier interpolated in between. The phonon density of states (DOS) is calculated by using the linear tetrahedral method with a total of 250 special q points in the irreducible wedge of the first BZ. To check the reliability of the pseudopotential plane-wave method for the fcc phase at very high pressure, phonon dispersion curves at selected directions are calculated with the all-electron linear-response FP-LMTO method. ${ }^{16}$ In this case, the dynamical matrix and phonon frequencies of Cs were obtained as a function of wave vector for a set of irreducible $\mathbf{q}$ points in a $(8,8,8)$ reciprocal lattice (29 points per 1/48th part of the BZ). The $3 \kappa-s p d$ LMTO basis with one-center expansions are performed inside the muffin tin (MT) spheres up to $l_{\max }=6$. In the interstitial region the basis functions are expanded in plane waves with the cutoff corresponding to the $28 \times 28 \times 28$ fast Fourier transform (FFT) grid in the unit cell of real space. The k-space integration needed for constructing the induced charge density is performed on a $16 \times 16 \times 16$ grid, which is twice as dense as the grid of phonon wave vectors $\mathbf{q}$.

\section{RESULTS}

Figure 1 shows the total energy per atom $E(V)$ as a function of volume for bcc and fcc Cs. The equilibrium atomic volumes corresponding to the minima in total energy are $97.05 \AA^{3}$ (bcc) and $100.89 \AA^{3}$ (fcc), respectively. The energy difference between bcc and fcc is only $4.1 \mathrm{meV} /$ atom. The small energy difference between the bcc phase and the fcc phase of Cs is quite similar to that of other alkali metals, e.g., lithium. ${ }^{20}$ The variation of the total energy with volume is also rather small, e.g., as the volume of bcc Cs changes from $97.05 \AA^{3}$ /atom to $80 \AA^{3} /$ atom $\left(\Delta V / V_{0}=-18 \%\right)$, the increase of the total energy is only about $25 \mathrm{meV}$, which means the Cs metal is a very soft material. The comparison of the pseudopotential calculations on the lattice constant, bulk modulus, and energy difference between bcc Cs and fcc Cs with that of FP-LAPW and experiments is shown in Table I. It can be seen in Table I that the results obtained by PS-PW calculations are in very good agreement with that of the all electron FP-LAPW calculations, which indicates that
TABLE I. The calculated lattice constant, bulk modulus, and energy difference between bcc and fcc of Cs using pseudopotential and FP-LAPW. The available experimental value (Ref. 21) at $4.2 \mathrm{~K}$ is also listed for comparison.

\begin{tabular}{clccc}
\hline \hline & & Pseudopotential & FP-LAPW & Experiment \\
\hline bcc & $a_{0}(\AA)$ & 5.79 & 5.78 & 5.92 \\
& $B_{0}(\mathrm{GPa})$ & 2.26 & 2.28 & 2.30 \\
fcc & $a_{0}(\AA)$ & 7.39 & 7.34 & \\
& $B_{0}(\mathrm{GPa})$ & 2.12 & 2.20 & \\
& $\Delta E^{\mathrm{fcc} / \mathrm{bcc}}$ & 4.1 & 5.0 & \\
& $(\mathrm{meV} / \mathrm{atom})$ & & & \\
& & & & \\
\end{tabular}

the interactions between the valence electrons and the atomic core are accurately described by the present pseudopotentials.

Next, we examine the phonon structures of the bcc and $\mathrm{fcc}^{(1)} \mathrm{Cs}$ at high pressures, where the bcc Cs has a higher Gibbs free energy than the close-packed $\mathrm{fcc}^{(1)}$ phase. It would be important to see if the phonons and elastic constants of the bcc Cs exhibit anomalies indicative of its dynamic instability at high pressures.

The PS-PW calculated phonon dispersion curves $[\nu(\mathbf{q})]$ of bcc Cs at several pressures are shown in Fig. 2, and the corresponding phonon DOS is shown in Fig. 3. Table II gives the elastic constants of the bcc and fcc Cs that are calculated from the slopes of the phonon dispersion curves at $\mathbf{q} \rightarrow 0$. The relationship between the elastic constant and the phonon dispersion is the following. At $\mathbf{q} \rightarrow 0, \rho v_{L}^{2}=C_{11}$,

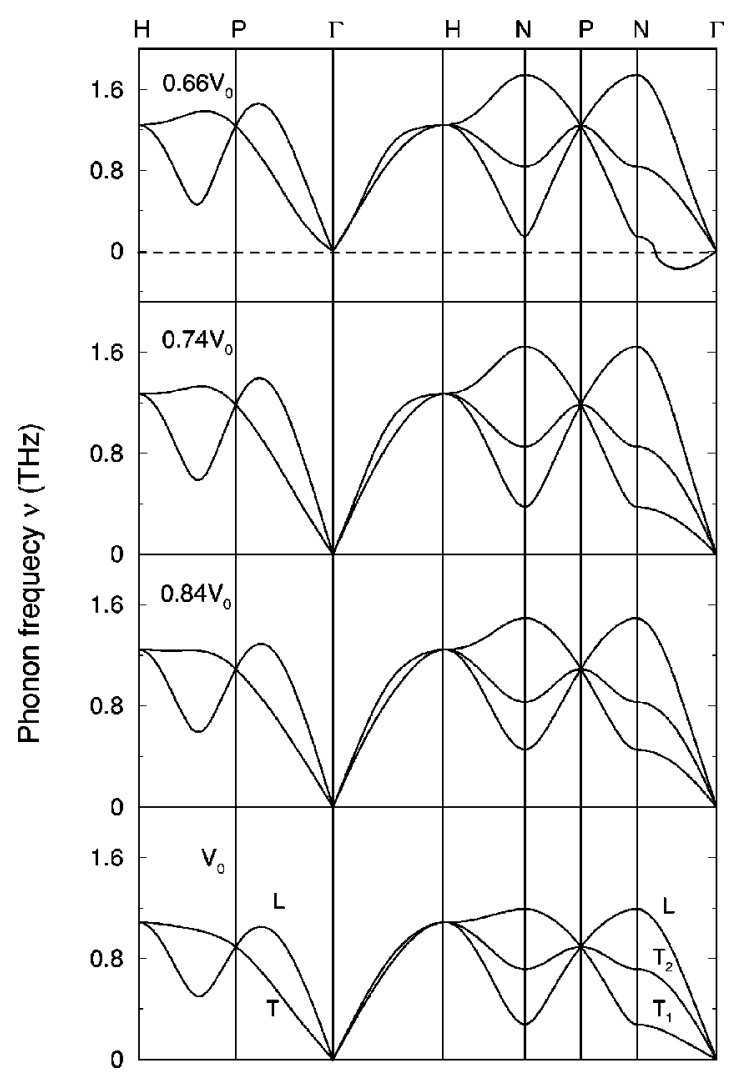

FIG. 2. The PS-PW calculated phonon frequencies of the bcc Cs at different volumes. 


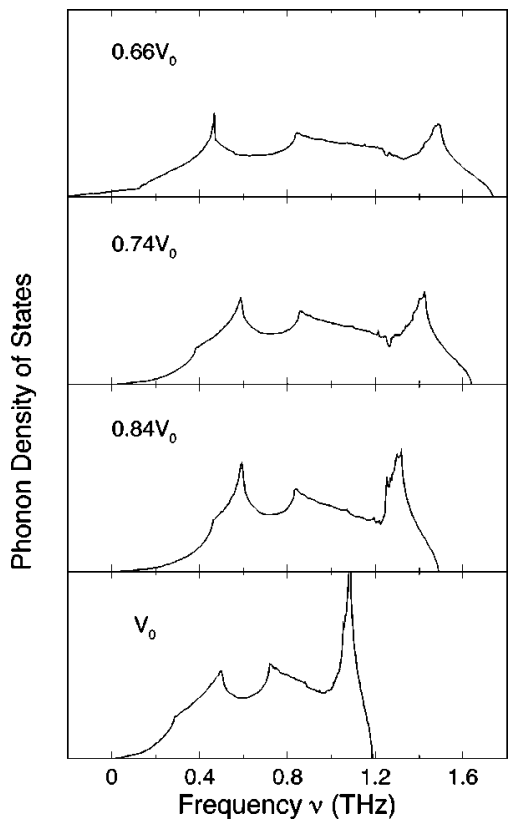

FIG. 3. The PS-PW calculated phonon DOS of the bcc Cs at different volumes.

$\rho v_{T}^{2}=C_{44}$ along [100] direction, where $\rho$ is the density of Cs, $v_{L}=d \omega_{L} / d \mathbf{q}, v_{T}=d \omega_{T} / d \mathbf{q}, \omega=2 \pi \nu$. Similarly, along [110] direction, $\rho v_{L}^{2}=\left(C_{11}+C_{12}\right) / 2+C_{44}, \quad \rho v_{T}^{2}=\left(C_{11}\right.$ $\left.-C_{12}\right) / 2, \rho v_{T^{\prime}}^{2}=C_{44}$. Along [111] direction, $\rho v_{L}^{2}=\left(C_{11}\right.$ $\left.+2 C_{12}+4 C_{44}\right) / 3, \rho v_{T}^{2}=\left(C_{11}-C_{12}+C_{44}\right) / 3$. Since the experimental measurements ${ }^{1,3}$ were performed at room temperature, the equilibrium volume of bcc Cs at zero pressure $V_{0}$ used in this paper is the theoretical value at $T=300 \mathrm{~K}$, which gives $V_{0}=102.43 \AA^{3}$. This theoretical equilibrium volume is calculated by minimizing the Helmholtz free energy of bec Cs at $T=300 \mathrm{~K}$ in the quasiharmonic approximation. We observed from Fig. 2 that the phonon frequencies of longitudinal modes (labeled by $L$ in Fig. 2) of the bcc Cs monotonically increase with pressure, while the phonon frequencies of the transverse modes along [ $\zeta \zeta 0]$ branch (labeled by $T_{1}$ ) first increase with pressure (for $V$ changes from $V_{0}$ to $0.84 V_{0}$ ) and then decrease with pressure (for $V$ changes from $0.74 V_{0}$ to $\left.0.66 V_{0}\right)$. When the volume of the bcc Cs is decreased to $0.66 V_{0}$, the tetragonal shear elastic constant $C^{\prime}=\frac{1}{2}\left(C_{11}-C_{12}\right)$ (in Table II) becomes negative. The long-wavelength transverse modes $\left(T_{1}\right)$ (in Fig. 2 ) be-

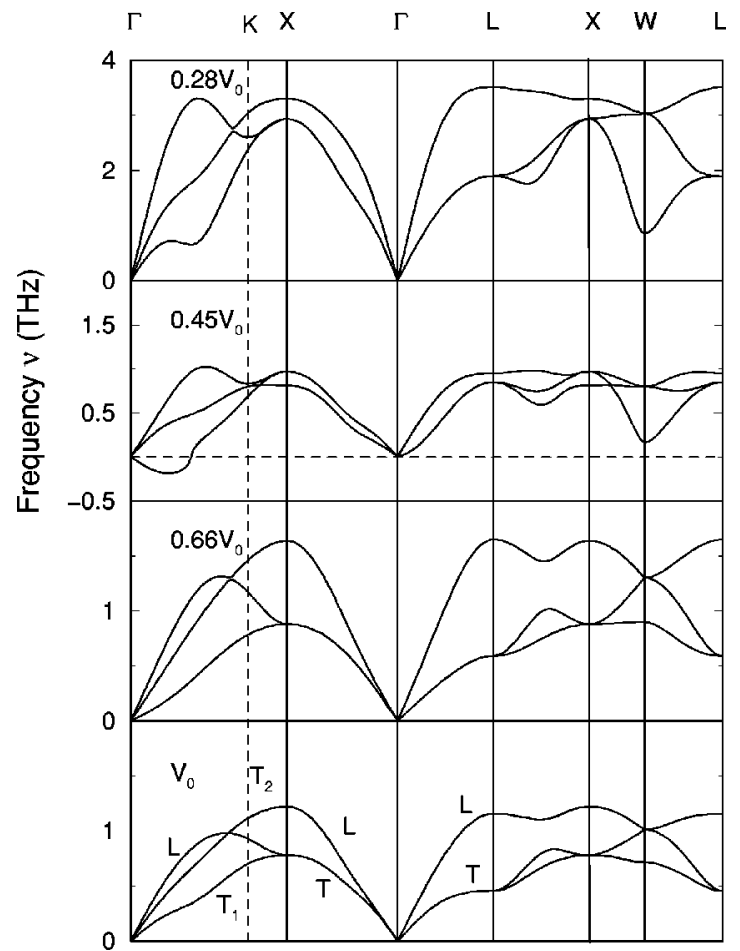

FIG. 4. The PW-PS calculated phonon frequencies of the fcc Cs at different volumes.

come unstable, where $-|\nu|$ is plotted when $\nu^{2}(\mathbf{q})<0$. At this pressure, the bcc Cs also has a higher enthalpy than fcc Cs (see Table II). Therefore, Cs is both dynamically and thermodynamically unstable at $V=0.66 V_{0}$. The calculated transition volume $V=0.68 V_{0}$ (when the $T_{1}$ modes are just becoming unstable) is in good agreement with the experimental value of $0.63 V_{0} .{ }^{1}$ The variation of phonon DOS with pressure (Fig. 3) reflects the changes of the phonon dispersion curves in Fig. 2. The high-frequency DOS peak shifts upwards in frequency with pressure, the low-frequency DOS peak first increases then decreases with pressure due to the softening of the $T_{1}$ modes. At the same time, the highfrequency peak becomes broader and lower at high pressure, while the low-frequency peak becomes sharper and higher at high pressure.

Figure 4 shows the phonon dispersion curves of the fcc $\mathrm{Cs}$ obtained from PS-PW calculations at different volumes. At $V=V_{0}$, fcc Cs is dynamically stable but the enthalpy is

TABLE II. The calculated elastic constants for bcc and fcc Cs. The available experimental data from Ref. 22 are indicated in parentheses.

\begin{tabular}{llcccc}
\hline \hline & & $V_{0}$ & $0.84 V_{0}$ & $0.74 V_{0}$ & $0.66 V_{0}$ \\
\hline bcc & $C_{11}(\mathrm{GPa})$ & $2.27(2.46)$ & 3.78 & 3.76 & 2.74 \\
& $C_{12}(\mathrm{GPa})$ & $1.88(2.06)$ & 1.94 & 2.44 & 3.67 \\
& $C_{44}(\mathrm{GPa})$ & $1.94(1.48)$ & 3.18 & 3.13 & 2.46 \\
& $C^{\prime}=\frac{1}{2}\left(C_{11}-C_{12}\right)(\mathrm{GPa})$ & $0.18(0.20)$ & 0.92 & 0.66 & -0.46 \\
$\mathrm{fcc}$ & $C_{11}(\mathrm{GPa})$ & 2.28 & 3.98 & 3.90 & 3.86 \\
& $C_{12}(\mathrm{GPa})$ & 1.88 & 3.24 & 2.87 & 3.18 \\
& $C_{44}(\mathrm{GPa})$ & 1.90 & 2.42 & 2.68 & 3.07 \\
& $C^{\prime}=\frac{1}{2}\left(C_{11}-C_{12}\right)(\mathrm{GPa})$ & 0.20 & 0.37 & 0.52 & 0.34 \\
& $\Delta H^{\mathrm{fcc} / \mathrm{bcc}}(\mathrm{meV} / \mathrm{atom})$ & 17.6 & 23.6 & 31.3 & -16.9 \\
\hline \hline
\end{tabular}



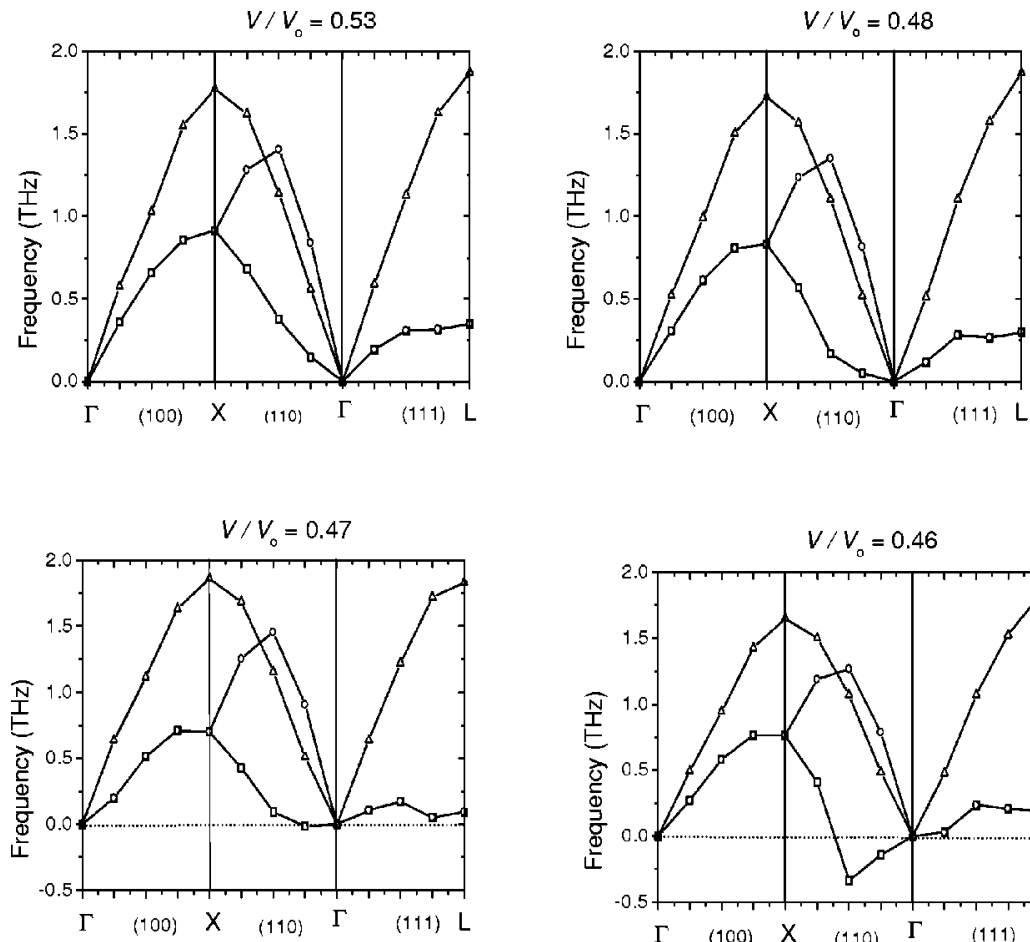

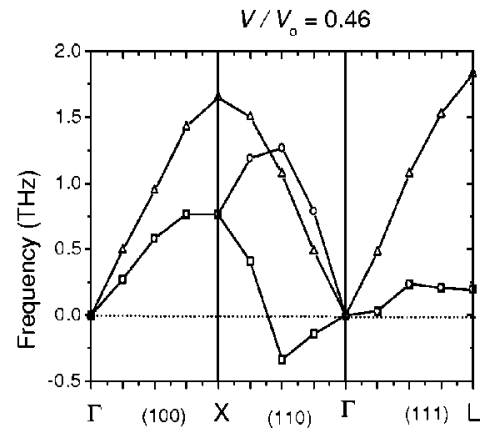

FIG. 5. Phonon frequencies of the fcc Cs at different volumes from FP-LMTO calculations. higher than the bcc phase. Therefore the $\mathrm{fcc}^{(1)} \mathrm{Cs}$ is thermodynamically metastable with respect to the bcc phase. At $V$ $=0.66 V_{0}$ where bcc becomes dynamically unstable, $\mathrm{fcc}^{(1)}$ is stable both dynamically (no soft modes) and thermodynamically $\left(\Delta H^{\mathrm{fcc} / \mathrm{bcc}}=H^{\mathrm{fcc}}-H^{\mathrm{bcc}}<0\right)$. That is the reason for the bcc $\mathrm{Cs}$ phase transition to the fcc $\mathrm{Cs}$ at this volume. When the pressure is increased even further $\left(V=0.46 V_{0}\right)$, the longwavelength $(\mathbf{q} \rightarrow 0)$ transverse modes along the $[\zeta \zeta 0]$ branch become unstable. This phonon softness may characterize the isostructural $\mathrm{fcc}^{(1)}-\mathrm{fcc}^{(2)}$ phase transition. Similar to the bcc-fcc ${ }^{(1)}$ phase transition at $V=0.66 V_{0}$ (Fig. 2), these unstable modes correspond to a negative elastic constant $C^{\prime}$. The softening of the acoustic phonon branch along [110] is associated with a mechanical instability that violates the Born stability condition ${ }^{23} \frac{1}{2}\left(C_{11}-C_{12}\right)>0$. However, there are significant different features of the phonon structure for the $f c c^{(1)}-\mathrm{fcc}^{(2)}$ transition from the bcc-fcc ${ }^{(1)}$ transition. At $V=0.45 V_{0}$, the phonon modes in the entire BZ become soft with respect to $V=0.66 V_{0}$. This interesting feature may characterize the isostructural $\mathrm{fcc}^{(1)}-\mathrm{fcc}^{(2)}$ phase transition of Cs. The same phonon instability is also observed with the FP-LMTO calculations. As shown in Fig. 5, in agreement with PS-PW calculations, the acoustic phonon frequencies along the [110] direction are predicted to become imaginary at $V=0.46 V_{0}$. The theoretical transition volume $V=0.46 V_{0}$ is in excellent agreement with the experimental value of $0.455 V_{0} \cdot^{3}$

The equations of state for the fcc phase computed from PS-PW and FP-LMTO methods are shown in Fig. 6. For both set of calculations there is a small change in the curvature near $V=48 \AA^{3}\left(0.47 V_{0}\right)$. This is followed by a plateau region where the pressure remains constant with decreasing volume. This plateau signifies the volume compression accompanying the isostructural transition. At smaller volume around $V=38 \AA^{3}$, there is a steep increase in the pressure indicating the onset of the stability region of the denser fcc ${ }^{(2)}$
(Cs III) phase. As shown in Fig. 4 , at $V=0.28 V_{0}$ the $\mathrm{fcc}^{(2)}$ $\mathrm{Cs}$ has recovered its stability. The estimated transition pressure from the FP-LMTO calculation is $5.2 \mathrm{GPa}$. This value is in substantial agreement with the experimental observation. ${ }^{3}$ The PS-PW calculations predicted a somewhat lower transition pressure. However, both calculations gave a significantly larger volume change $(\approx-20 \%)$ than the experimental value of $-9 \%$.

Figure 7 shows the phonon DOS of the fcc Cs calculated by PS-PW at different volumes. It can be seen that when the volume changes from $V_{0}$ to $0.66 V_{0}$, the phonon frequency shifts upward. When the volume is further decreased to $0.45 V_{0}$ where the $\mathrm{fcc}^{(1)}$-to-fcc ${ }^{(2)}$ phase transition occurs, there are significant softening for all the phonon modes (downward shift of the phonon frequencies). Some states appear at $\nu<0$ representing the unstable phonon modes along $[\zeta \zeta 0]$ branch. The phonon softening for all the phonon modes reflects the collapse of the $f_{c c}{ }^{(1)}$ structure. When the volume is further decreased to $0.28 V_{0}$, there are no unstable

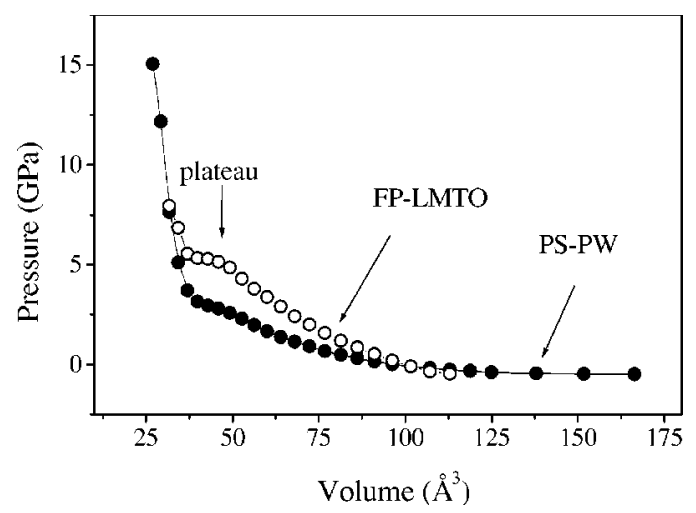

FIG. 6. Calculated equation of state using PS-PW and FPLMTO methods. 


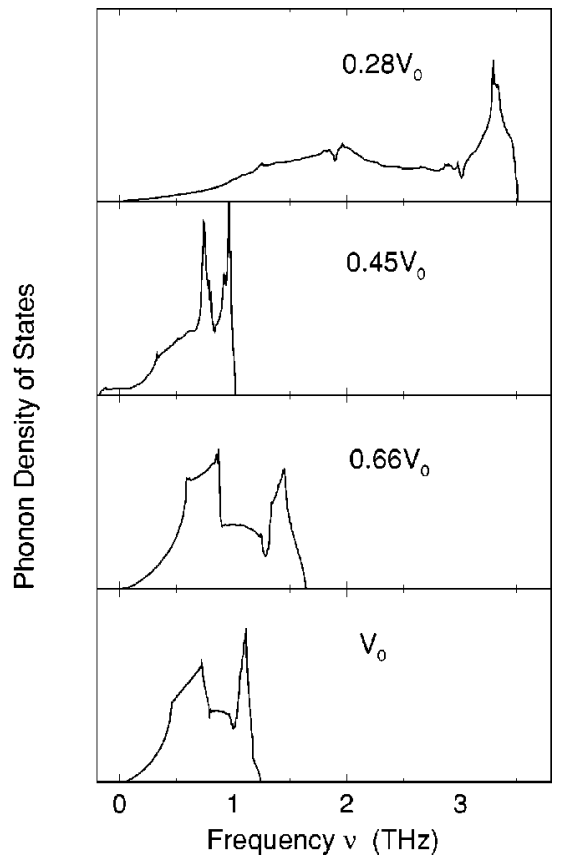

FIG. 7. PS-PW calculated phonon DOS of fcc Cs at different volumes.

modes indicating the completion of the isostructural phase transition.

It is known that the $\mathrm{bcc} \rightarrow \mathrm{fcc}$ transition in high-pressure $\mathrm{Cs}$ is due to a $s \rightarrow d$ hybridization of the valence band. Figure 8 shows the FP-LAPW calculated charge density on the (100) plane of fcc Cs at (a) $V=V_{0}$ and (b) $V=0.39 V_{0}$. It is very clear that the interactions between $C$ s atoms have changed from majority $s$-like orbitals to $d$-type orbitals when $\mathrm{Cs}$ is compressed to a small volume. The theoretical calculations confirm the expected results but also reveal the physical basis of this electronic transition. At high pressure, $s$-orbital interactions become energetically unfavorable. The transition from $s$ orbitals to $d$ orbitals helps to alleviate the electron repulsion and decreases the kinetic energy of the valence electrons. Consequently, the electrons become more localized and the interactions between $\mathrm{Cs}$ atoms become more covalent. This observation has a very important implication. The increased covalency indicates a more directional structure. Therefore, under very high pressure, dense packing may not be the most favorable structure and other more “open" structures such as that in Cs IV (Ref. 6) and V (Ref. 7) are preferred. This may be a general phenomenon for (a)

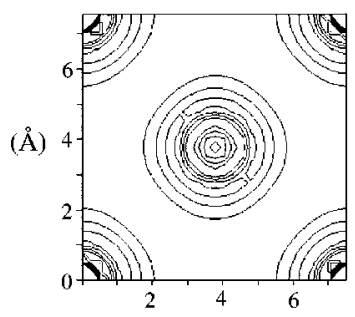

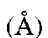

(b)

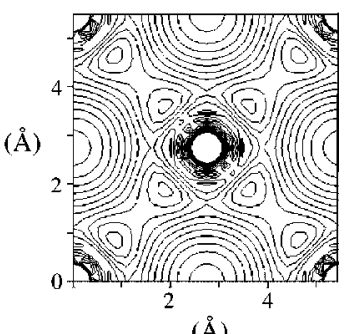

(A)
FIG. 8. FP-LAPW calculated charge densities along the [100] plane of fcc Cs at (a) $V=V_{0}$, and (b) $V=0.39 V_{0}$.

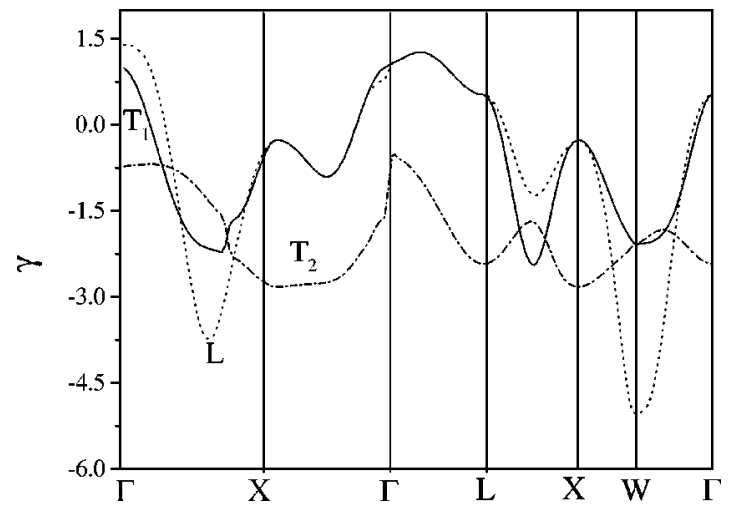

FIG. 9. Calculated dispersion curves of the mode Grüneisen parameters of $\mathrm{Cs}$ along indicated symmetry lines in the BZ corresponding to a volume of $49.3 \AA^{3}$. The solid, dashed and dotteddashed lines are for the two transverse and longitudinal modes, respectively.

simple metals and alloys at high pressure. A similar effect was observed in a previous study on the structures of $\mathrm{K}-\mathrm{Ag}$ alloys. $^{24}$

The calculated dispersion curves of the mode Grüneisen parameters for $\mathrm{fcc}^{(1)} \mathrm{Cs}$ near the phase transition volume ( $V=0.48 V_{0}$ ) obtained by the PS-PW are depicted in Fig. 9 . It is shown that the Grüneisen parameters along several directions in the $\mathrm{BZ}$ become negative, which indicates that the phonon frequencies are decreasing with compression in these regions. To investigate the physical implications of this behavior, the average Grüneisen parameter $\gamma$ was obtained by integration over the dispersion curves of the mode Gruneisen parameters. It is interesting to observe that the average Grüneisen parameter decreases when Cs is compressed (Fig. 10). It becomes negative as the volume was compressed to the range near the $\mathrm{fcc}^{(1)}$ to $\mathrm{fcc}^{(2)}$ transition and jumps to positive values once the phase transition is finished. This behavior is in agreement with the experimental measurements ${ }^{25}$ and is exactly the same as has been suggested from a phenomenological model. ${ }^{26}$ The magnitude of $\gamma$ and its variation with the volume from the present first-principles calculations are in very good agreement with the estimate from the phenomenological approach ${ }^{26}$ with a minor exception that the range

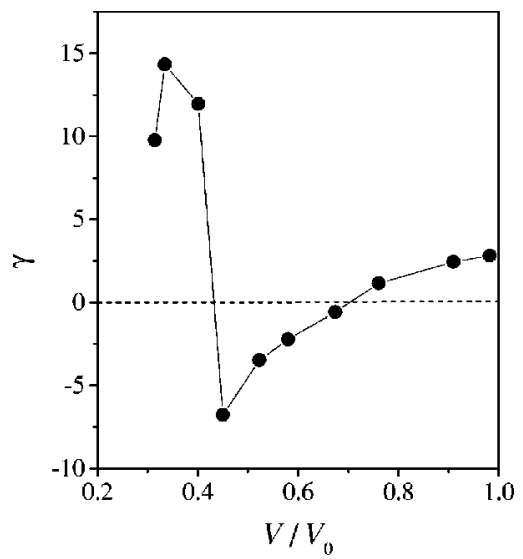

FIG. 10. Averaged Grüneisen parameter $\gamma$ for fcc Cs as a function of volume. The negative values of $\gamma$ indicate the region where the phonon frequencies are decreasing. 
where the average Grüneisen parameters are negative is somewhat broader. The free energy of the system can be computed within the quasiharmonic approximation using the calculated mode Grüneisen parameters. ${ }^{26,27}$ The result indicates a strong temperature dependence for the isostructural phase transition. This conclusion is in exact accord with the suggestion from the previous phenomenological model.

\section{CONCLUSION}

In conclusion, we have studied the phonon instabilities of the bcc and the fcc Cs during its high pressure phase transitions. It is found that at low pressure, both the bcc Cs and fcc Cs are dynamically stable but the fcc Cs is thermodynami- cally metastable (has a higher enthalpy than bcc Cs). The high-pressure phase transitions of $\mathrm{Cs}$ from bcc to $\mathrm{fcc}^{(1)}$ and from $\mathrm{fcc}^{(1)}$ to another $\mathrm{fcc}^{(2)}$ are related to the phonon instabilities of the long-wavelength transverse acoustic modes. It is interesting to find that during the $\mathrm{fcc}^{(1)}-\mathrm{fcc}^{(2)}$ isostructural phase transition, the phonon modes in the entire BZ become soft.

\section{ACKNOWLEDGMENTS}

This work was supported by the Department of Energy (DOE) under Contract No. W-7405-ENG-36. The authors thank Stefano de Gironcoli and Stefano Baroni for providing the pseudopotential of Cs.
${ }^{1}$ P.W. Bridgman, Proc. Am. Acad. Arts Sci. 76, 55 (1948).

${ }^{2}$ G.C. Kennedy, A. Jayaman, and R.C. Newton, Phys. Rev. 126, 1363 (1962).

${ }^{3}$ H.T. Hall, L. Merrill, and J.D. Barnett, Science 146, 1297 (1964).

${ }^{4}$ R.A. Stager and H.G. Drickamer, Phys. Rev. Lett. 12, 19 (1964).

${ }^{5}$ A. Jayaraman, R.C. Newton, and J.M. McDonough, Phys. Rev. 159, 527 (1967).

${ }^{6}$ K. Takemura, S. Minomura, and O. Shimomura, Phys. Rev. Lett. 49, 1772 (1982).

${ }^{7}$ U. Schwarz, K. Takemura, M. Hanfland, and K. Syassen, Phys. Rev. Lett. 81, 2711 (1998).

${ }^{8}$ S.G. Louie and M.L. Cohen, Phys. Rev. B 10, 3237 (1974).

${ }^{9}$ A.K. McMahan, Phys. Rev. B 29, 5982 (1984).

${ }^{10}$ S. Carlesi, A. Franchini, V. Bortolani, and S. Martinelli, Phys. Rev. B 59, 11716 (1999).

${ }^{11}$ M.B. Nardelli, S. Baroni, and P. Giannozzi, Phys. Rev. B 51, 8060 (1995).

${ }^{12}$ A.M. Saitta, D. Alfe, S. de Gironcoli, and S. Baroni, Phys. Rev. Lett. 78, 4958 (1997).

${ }^{13}$ K.M. Ho, C.L. Fu, and B.N. Harmon, Phys. Rev. B 28, 6687 (1983).

${ }^{14}$ K. Einarsdotter, B. Sadigh, G. Girmvall, and V. Ozolins, Phys. Rev. Lett. 79, 2073 (1997).

${ }^{15}$ P. Blaha, K. Schwarz, and J. Luitz, in wIEN97, A Full Potential
Linearized Augmented Plane Wave Package for Calculating Crystal Properties, edited by Karlheinz Schwarz (Techn. Univ. Wien, Vienna 1999). Updated version of P. Blaha, K. Schwarz, P. Sorantin, and S.B. Trickey, Comput. Phys. Commun. 59, 399 (1990).

${ }^{16}$ S.Y. Savrasov, Phys. Rev. Lett. 69, 2819 (1992).

${ }^{17}$ M. Methfessel and A.T. Paxton, Phys. Rev. B 40, 3616 (1989).

${ }^{18}$ H.J. Monkhorst and J.D. Pack, Phys. Rev. B 13, 5188 (1976).

${ }^{19}$ S. Baroni, P. Giannozzi, and A. Testa, Phys. Rev. Lett. 58, 1861 (1987); P. Giannozzi, S. de Gironcoli, P. Pavone, and S. Baroni, Phys. Rev. B 43, 7231 (1991).

${ }^{20}$ M. Hanfland, I. Loa, K. Syassen, U. Schwarz, and K. Takemura, Solid State Commun. 112, 123 (1999).

${ }^{21}$ C.A. Swenson, Phys. Rev. 99, 423 (1955).

${ }^{22}$ F.J. Kollarits and J. Trivison, J. Phys. Chem. Solids 29, 2133 (1968).

${ }^{23}$ M. Born and K. Huang, Dynamical Theory of Crystal Lattices (Clarendon Press, Oxford, 1954).

${ }^{24}$ J.S. Tse, G. Frapper, A. Ker, R. Rousseau, and D.D. Klug, Phys. Rev. Lett. 82, 4472 (1999).

${ }^{25}$ R. Boehler and M. Ross, Phys. Rev. B 29, 3673 (1984).

${ }^{26}$ D. Glotzel and A.K. McMahan, Phys. Rev. B 20, 3210 (1979).

${ }^{27}$ J. Xie, S. de Gironcoli, S. Baroni, and M. Scheffler, Phys. Rev. B 59, 965 (1999). 